\title{
Characterization of magnetic tunnel junction test pads
}

Østerberg, Frederik Westergaard; Kjær, Daniel; Nielsen, Peter Folmer; Hansen, Ole; Petersen, Dirch Hjorth

Published in:

Journal of Applied Physics

Link to article, DOI:

$10.1063 / 1.4932663$

Publication date:

2015

Document Version

Publisher's PDF, also known as Version of record

Link back to DTU Orbit

Citation $(A P A)$ :

Østerberg, F. W., Kjær, D., Nielsen, P. F., Hansen, O., \& Petersen, D. H. (2015). Characterization of magnetic tunnel junction test pads. Journal of Applied Physics, 118(14), 143901. https://doi.org/10.1063/1.4932663

\section{General rights}

Copyright and moral rights for the publications made accessible in the public portal are retained by the authors and/or other copyright owners and it is a condition of accessing publications that users recognise and abide by the legal requirements associated with these rights.

- Users may download and print one copy of any publication from the public portal for the purpose of private study or research.

- You may not further distribute the material or use it for any profit-making activity or commercial gain

- You may freely distribute the URL identifying the publication in the public portal

If you believe that this document breaches copyright please contact us providing details, and we will remove access to the work immediately and investigate your claim. 


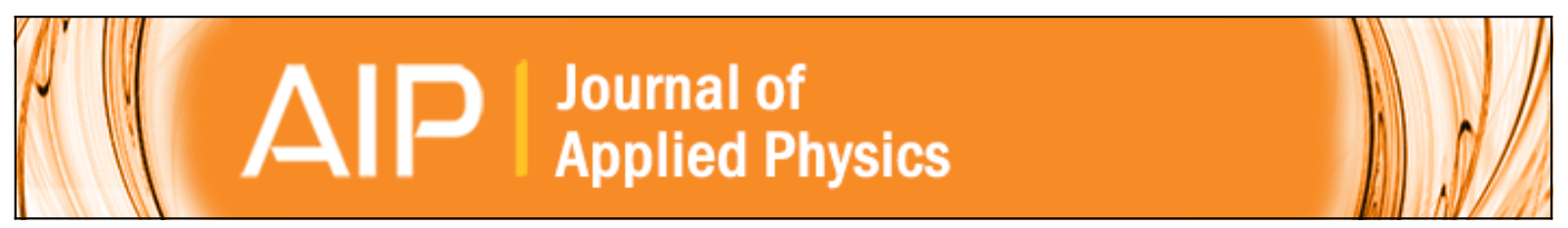

\section{Characterization of magnetic tunnel junction test pads}

Frederik Westergaard Østerberg, Daniel Kjær, Peter Folmer Nielsen, Ole Hansen, and Dirch Hjorth Petersen

Citation: Journal of Applied Physics 118, 143901 (2015); doi: 10.1063/1.4932663

View online: http://dx.doi.org/10.1063/1.4932663

View Table of Contents: http://scitation.aip.org/content/aip/journal/jap/118/14?ver=pdfcov

Published by the AIP Publishing

\section{Articles you may be interested in}

The effect of the static magnetic susceptibility on the spin precession in $\mathrm{MgO}$ based magnetic tunnel junction Appl. Phys. Lett. 98, 242509 (2011); 10.1063/1.3601463

On-film tunneling resistance measurements of unpatterned magnetic tunnel junctions

J. Appl. Phys. 108, 093902 (2010); 10.1063/1.3503375

Direct communication between magnetic tunnel junctions for nonvolatile logic fan-out architecture Appl. Phys. Lett. 97, 152504 (2010); 10.1063/1.3499427

rf amplification in a three-terminal magnetic tunnel junction with a magnetic vortex structure Appl. Phys. Lett. 95, 022513 (2009); 10.1063/1.3177188

$1 / \mathrm{f}$ noise in linearized low resistance $\mathrm{MgO}$ magnetic tunnel junctions

J. Appl. Phys. 99, 08B314 (2006); 10.1063/1.2172179

\section{AlP $\left.\right|_{\text {APL Photonics }}$}

APL Photonics is pleased to announce Benjamin Eggleton as its Editor-in-Chief

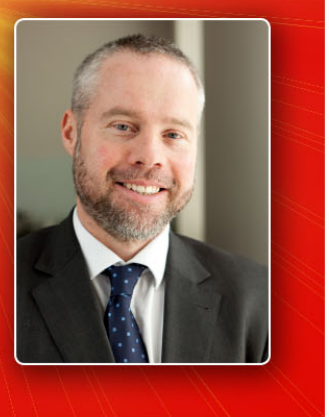




\title{
Characterization of magnetic tunnel junction test pads
}

\author{
Frederik Westergaard Østerberg, ${ }^{1, a)}$ Daniel Kjær, ${ }^{1,2}$ Peter Folmer Nielsen, ${ }^{2}$ Ole Hansen, ${ }^{1,3}$ \\ and Dirch Hjorth Petersen ${ }^{1, b)}$ \\ ${ }^{1}$ Department of Micro- and Nanotechnology, Technical University of Denmark, DTU Nanotech, \\ Building 345 East, DK-2800 Kongens Lyngby, Denmark \\ ${ }^{2}$ CAPRES A/S, Scion-DTU, Building 373, DK-2800 Kongens Lyngby, Denmark \\ ${ }^{3}$ CINF-Centre for Individual Nanoparticle Functionality, Technical University of Denmark, \\ Building 345 East, DK-2800 Kongens Lyngby, Denmark
}

(Received 3 July 2015; accepted 28 September 2015; published online 12 October 2015)

\begin{abstract}
We show experimentally as well as theoretically that patterned magnetic tunnel junctions can be characterized using the current-in-plane tunneling (CIPT) method, and the key parameters, the resistance-area product (RA) and the tunnel magnetoresistance (TMR), can be determined. The CIPT method relies on four-point probe measurements performed with a range of different probe pitches and was originally developed for infinite samples. Using the method of images, we derive a modified CIPT model, which compensates for the insulating boundaries of a finite rectangular sample geometry. We measure on square tunnel junction pads with varying sizes and analyze the measured data using both the original and the modified CIPT model. Thus, we determine in which sample size range the modified CIPT model is needed to ensure validity of the extracted sample parameters, RA and TMR. In addition, measurements as a function of position on a square tunnel junction pad are used to investigate the sensitivity of the measurement results to probe misalignment. (C) 2015 AIP Publishing LLC. [http://dx.doi.org/10.1063/1.4932663]
\end{abstract}

\section{INTRODUCTION}

Since their discovery by Jullière, ${ }^{1}$ magnetic tunnel junctions (MTJ) have attracted considerable interest due to the multitude of applications as sensors, ${ }^{2}$ read heads in hard disc drives, ${ }^{3-7}$ and, in particular, their use in magnetoresistive random-access memory (MRAM). ${ }^{4,8-10}$ MRAM has the potential to become the preferred memory technology of the future, due to the outstanding technical performance, such as high speed, high density, non-volatility, reliability, and very low power consumption. ${ }^{4,9,11}$

Traditionally, a MTJ consists of two ferromagnetic layers separated by a thin tunnel barrier layer; often one ferromagnetic layer is pinned while the other is "free," i.e., has a much lower switching magnetic field. As a result, the MTJ can assume two states, where the magnetization of the ferromagnetic layers are either parallel or anti-parallel, corresponding to low or high tunnel resistance; often characterized by the corresponding resistance-area products $\mathrm{RA}_{\text {low }}$ and $\mathrm{RA}_{\text {high, }}{ }^{12}$ respectively. The contrast between $\mathrm{RA}_{\text {low }}$ and $\mathrm{RA}_{\text {high }}$ is the tunnel magnetoresistance $(\mathrm{TMR})$, i.e., $\quad \mathrm{TMR}=\left(\mathrm{RA}_{\text {high }}\right.$ $\left.-\mathrm{RA}_{\text {low }}\right) / \mathrm{RA}_{\text {low }}$, which is of particular importance for MRAM applications. These key parameters can either be measured on final devices or on complete films prior to device fabrication using the current-in-plane tunneling (CIPT) method. ${ }^{12}$ A distinct advantage of the CIPT method is that $\mathrm{TMR}$ and $\mathrm{RA} \equiv \mathrm{RA}_{\text {low }}$ can be determined without performing lithography, etching and inter-connections to the top and

\footnotetext{
${ }^{\text {a)} E l e c t r o n i c ~ m a i l: ~ F r e d e r i k . O s t e r b e r g @ n a n o t e c h . d t u . d k ~}$

${ }^{b)}$ Electronic mail: Dirch.Petersen@nanotech.dtu.dk
}

bottom electrodes of the MTJ, and thereby information about TMR and resistance-area product (RA) can be obtained at an earlier stage than that of the final device. On the other hand, one of the disadvantages of the current CIPT method is that it requires an "infinite" sample, which means it is not possible to monitor whether the processes following the deposition of the MTJ stack influence the TMR and RA values.

CIPT measurements on actual device wafers have to be performed on test pads that potentially can be placed in scribe lines to save area. Here, we investigate how the insulating boundaries of patterned square test pads affect CIPT measurements when compared to measurements on a full film. The original theory for the CIPT method was derived assuming infinite samples. Here, we use the method of images ${ }^{13,14}$ to derive a modified model which is valid for finite samples of rectangular shape.

\section{THEORY}

CIPT measurements can be performed using a colinear multi-point probe, where four-point sub-probes are used for individual measurements. Four-point probe measurements may be done in several different configurations, and here configurations A and B are of interest. In configuration A, the two outer pins are used as current source and drain while the two inner pins used for measuring the voltage drop as sketched in Fig. 1. For configuration B, the roles of pins 3 and 4 are interchanged compared to configuration A as shown in Fig. 1.

Worledge and Trouilloud ${ }^{12}$ model the MTJ film as two infinite, thin conducting sheets of sheet resistances $R_{\mathrm{t}}$ (top) and $R_{\mathrm{b}}$ (bottom) with a connecting interface specific contact 


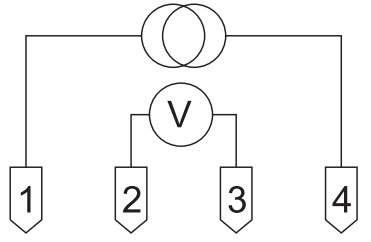

A

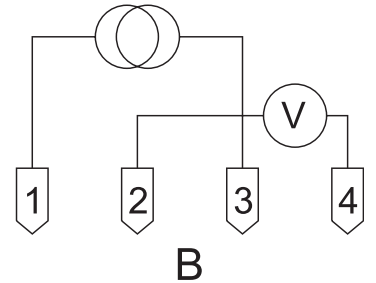

FIG. 1. The probe configurations A and B used for CIPT measurements.

resistance RA. The potential $\Phi$ at the point $\mathbf{r}$ from a single current source $I_{0}$ placed at $\mathbf{r}_{0}$ on an infinite MTJ film can be written as ${ }^{15}$

$$
\Phi\left(\mathbf{r}, \mathbf{r}_{0}\right)=\frac{I_{0} R_{\|}}{2 \pi}\left\{\frac{R_{\mathrm{t}}}{R_{\mathrm{b}}} \mathrm{K}_{0}\left(\frac{\left|\mathbf{r}-\mathbf{r}_{0}\right|}{\lambda}\right)-\ln \left(\frac{\left|\mathbf{r}-\mathbf{r}_{0}\right|}{\lambda}\right)\right\},
$$

where $\mathrm{K}_{0}$ is the modified Bessel function of the second kind of order 0 and $R_{\|}$is the sheet resistance of the two sheets in parallel

$$
R_{\|}=\frac{R_{\mathrm{t}} R_{\mathrm{b}}}{R_{\mathrm{t}}+R_{\mathrm{b}}}
$$

while $\lambda$ is the transfer length, a characteristic sample length scale, obtained from

$$
\lambda=\sqrt{\frac{\mathrm{RA}}{R_{\mathrm{t}}+R_{\mathrm{b}}}} .
$$

In Eq. (1), the logarithmic term is reminiscent of the expression for a single sheet, while the Bessel term is the correction needed due to the finite specific contact resistance between the sheets.

For a four-point probe on an infinite sample with the current source $I_{0}$ placed at $\mathbf{r}_{i}$ and drain at $\mathbf{r}_{j}$ and the voltage probes at $\mathbf{r}_{k}$ and $\mathbf{r}_{l}$, the four-point resistance can be calculated from Eq. (1) using super-position

$$
R=\frac{\Phi\left(\mathbf{r}_{k}, \mathbf{r}_{i}\right)-\Phi\left(\mathbf{r}_{l}, \mathbf{r}_{i}\right)-\Phi\left(\mathbf{r}_{k}, \mathbf{r}_{j}\right)+\Phi\left(\mathbf{r}_{l}, \mathbf{r}_{j}\right)}{I_{0}} .
$$

If the four probe pins are equally spaced with the pin spacing $s$, the expected resistances for CIPT measurements on an infinite sample in probe configurations $\mathrm{A}$ and $\mathrm{B}$ are

$$
R_{\mathrm{A}_{\mathrm{inf}}}=\frac{R_{\|}}{2 \pi}\left\{\frac{R_{\mathrm{t}}}{R_{\mathrm{b}}}\left[2 \mathrm{~K}_{0}\left(\frac{s}{\lambda}\right)-2 \mathrm{~K}_{0}\left(\frac{2 s}{\lambda}\right)\right]+\ln (4)\right\},
$$

$$
R_{\mathrm{B}_{\mathrm{inf}}}=\frac{R_{\|}}{2 \pi}\left\{\frac{R_{\mathrm{t}}}{R_{\mathrm{b}}}\left[\mathrm{K}_{0}\left(\frac{s}{\lambda}\right)-\mathrm{K}_{0}\left(\frac{3 s}{\lambda}\right)\right]+\ln (3)\right\}
$$

respectively.

However, in real measurements, the probe pins will not land exactly where expected; each electrode will have some position error as described by Kjaer et al. ${ }^{16}$ For single sheet micro four-point probe sheet resistance measurements, it has previously $^{16}$ been shown that Van der Pauw's method ${ }^{17,18}$ effectively reduces the effect of pin position errors. In Van der Pauw's method, a pseudo sheet resistance $R_{\mathrm{P}}$ is defined by the equation

$$
\exp \frac{2 \pi R_{\mathrm{A}}}{R_{\mathrm{P}}}-\exp \frac{2 \pi R_{\mathrm{B}}}{R_{\mathrm{P}}}=1 .
$$

In the case of a single infinite sheet, $R_{\mathrm{P}}$ is identical to the sheet resistance; on a MTJ, $R_{\mathrm{P}}$ does not have a direct physical interpretation, but it proves useful anyway and is less affected by pin position errors than both $R_{\mathrm{A}}$ and $R_{\mathrm{B}}$ even though perfect error cancellation cannot be expected.

\section{A. Rectangular samples}

For measurements in vicinity of an insulating boundary, the theory derived for an infinite sample is no longer valid, since the current density normal to the boundary must be zero. In order to fulfil this boundary condition, the method of images is applied. ${ }^{13,14}$ In practice, this means that additional current sources and drains are placed at the positions obtained by mirroring the current sources and drains in the lines defining the boundaries as shown in Fig. 2. Mirror images of the additional current sources and drains are also needed.

Figure 2 shows a sketch of a rectangular sample with dimensions of length $l$ and width $w$; the insulating boundaries are represented by solid black lines. The original current source and drain are labelled $I_{+}$and $I_{-}$, respectively. The center of the four-point probe is marked by an $\times$ and is positioned at $\left(x_{\mathrm{c}}, y_{\mathrm{c}}\right)$. For practical reasons, only the mirror images closest to the pad are shown.

The potential for a rectangle, $0 \leq y \leq w, 0 \leq x \leq l$, with insulating boundaries at $y=0, y=w, x=0$, and $x=l$ can be written as a double infinite sum, since the images of the source or drain are positioned at $\pm \mathbf{r}_{0}+2(n \mathbf{w}+m \mathbf{l})$ and $\pm \overline{\mathbf{r}_{0}}+2(n \mathbf{w}+m \mathbf{l})$. Here, $n$ and $m$ are summation integers and the vectors are defined as $\mathbf{w}=w \mathbf{e}_{y}$ and $\mathbf{l}=l \mathbf{e}_{x}$; the vector $\mathbf{r}_{0}=\left(x_{0}, y_{0}\right)$ is the position of the source or drain, while $\overline{\mathbf{r}_{0}}=\left(x_{0},-y_{0}\right)$. As a result, the potential becomes

$$
\begin{aligned}
\Phi\left(\mathbf{r}, \mathbf{r}_{0}\right)= & I_{0} \frac{R_{\|}}{2 \pi} \sum_{n=-\infty}^{\infty} \sum_{m=-\infty}^{\infty}\left\{\frac { R _ { \mathrm { t } } } { R _ { \mathrm { b } } } \left[\mathrm{K}_{0}\left(\frac{\left|\mathbf{r}-\mathbf{r}_{0}-2(n \mathbf{w}+m \mathbf{l})\right|}{\lambda}\right)+\mathrm{K}_{0}\left(\frac{\left|\mathbf{r}-\overline{\mathbf{r}_{0}}-2(n \mathbf{w}+m \mathbf{l})\right|}{\lambda}\right)\right.\right. \\
& \left.+\mathrm{K}_{0}\left(\frac{\left|\mathbf{r}+\mathbf{r}_{0}-2(n \mathbf{w}+m \mathbf{l})\right|}{\lambda}\right)+\mathrm{K}_{0}\left(\frac{\left|\mathbf{r}+\overline{\mathbf{r}_{0}}-2(n \mathbf{w}+m \mathbf{l})\right|}{\lambda}\right)\right]-\ln \left(\frac{\left|\mathbf{r}-\mathbf{r}_{0}-2(n \mathbf{w}+m \mathbf{l})\right|}{\lambda}\right) \\
& \left.-\ln \left(\frac{\left|\mathbf{r}-\overline{\mathbf{r}_{0}}-2(n \mathbf{w}+m \mathbf{l})\right|}{\lambda}\right)-\ln \left(\frac{\left|\mathbf{r}+\mathbf{r}_{0}-2(n \mathbf{w}+m \mathbf{l})\right|}{\lambda}\right)-\ln \left(\frac{\left|\mathbf{r}+\overline{\mathbf{r}_{0}}-2(n \mathbf{w}+m \mathbf{l})\right|}{\lambda}\right)\right\} .
\end{aligned}
$$




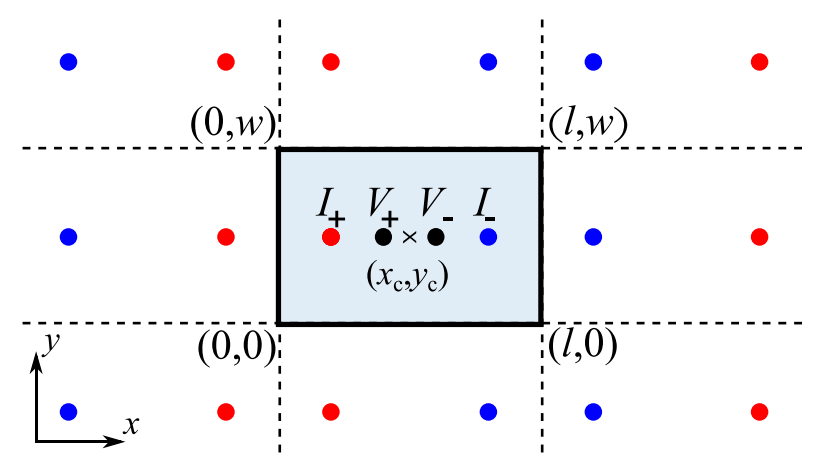

FIG. 2. Sketch of a rectangular shaped sample of length $l$ and width $w$ (light blue) with insulating boundaries (solid black lines). A four-point probe is placed on the sample, with the center of the probe at $\left(x_{\mathrm{c}}, y_{\mathrm{c}}\right)$. The eight closest mirror images of both the current source and drain are also shown.

By inserting Eq. (8) into Eq. (4), the four-point resistances for a rectangular sample with pin configuration $\mathrm{A}$ $\left(R_{\mathrm{A}, \text { rect }}\right)$ and pin configuration $\mathrm{B}\left(R_{\mathrm{B}, \text { rect }}\right)$ can be calculated. These values can then be inserted into Eq. (7) to obtain the pseudo sheet resistance for a rectangular sample $\left(R_{\mathrm{P}, \text { rect }}\right)$.

In the calculations, the double infinite sums in Eq. (8) are truncated to include only the terms $|n| \leq 7$ and $|m| \leq 7$ since higher index terms are below the numeric noise.

\section{B. Resistances on square pads and infinite samples}

The impact of insulating boundaries on the resistances $R_{\mathrm{A}}, R_{\mathrm{B}}$, and $R_{\mathrm{P}}$ can be illustrated by the relative deviation, $\left(R_{\mathrm{sq}}-R_{\text {inf }}\right) / R_{\text {inf }}$, between resistances calculated for a square sample $\left(R_{\text {sq }}\right)$ and those of an infinite sample $\left(R_{\text {inf }}\right)$ with otherwise identical parameters. In Fig. 3, the relative deviations for $R_{\mathrm{A}}, R_{\mathrm{B}}$, and $R_{\mathrm{P}}$ are shown as a function of the normalized width $(w / s)$ of the square. The sample parameters used in the calculation were $R_{\|}=1 \Omega, R_{\mathrm{t}} / R_{\mathrm{b}}=1$, and $\lambda=1 \mu \mathrm{m}$, while the probe pitch was $s=\lambda$. Additional calculations for $\lambda=0.1 \mu \mathrm{m}$ and $\lambda=10 \mu \mathrm{m}$ illustrate how the relative deviation for $R_{\mathrm{P}}$ changes with $\lambda$. In Appendix $\mathrm{A}$, contour plots

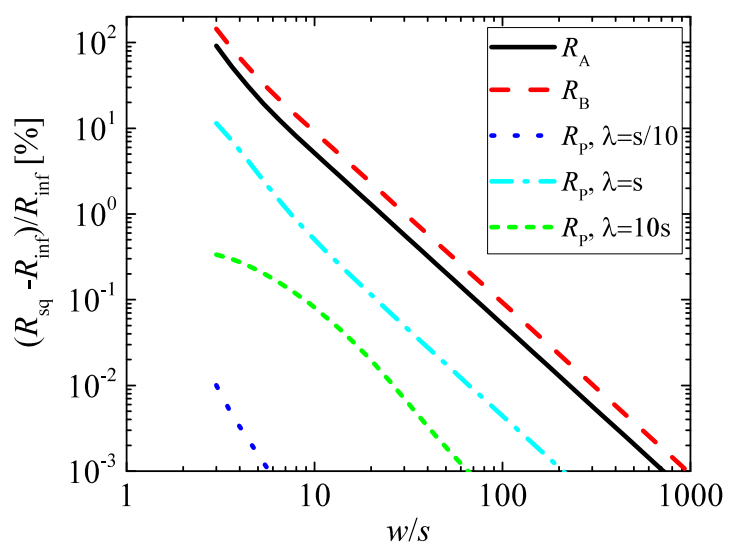

FIG. 3. The relative deviation $\left(R_{\mathrm{sq}}-R_{\text {inf }}\right) / R_{\text {inf }}$ between calculated fourpoint resistances for square samples $\left(R_{\mathrm{sq}}\right)$ compared to calculated resistances for an infinite sample $\left(R_{\text {inf }}\right)$ as function of normalized square size $w / s$. Deviations for $R_{\mathrm{A}}, R_{\mathrm{B}}$, and $R_{\mathrm{P}}$ are shown. The resistances for the squares were calculated assuming a four-point probe placed at the centre of the pad and parallel to an edge of the pad. show in more detail how the relative deviation of $R_{\mathrm{P}}$ depends on $w / s, \lambda / s$, and $R_{\mathrm{t}} / R_{\mathrm{b}}$.

Figure 3 shows that the resistances on the square sample are always larger than those on the infinite sample for a probe placed at the centre of a pad with the electrodes parallel to two of the boundaries. The relative deviation of the resistances are seen to decrease rapidly with increasing size of the square, approximately according to $(w / s)^{-2}$. Importantly, the relative deviation of $R_{\mathrm{P}}$ is approximately an order of magnitude smaller than those of $R_{\mathrm{A}}$ and $R_{\mathrm{B}}$ for $\lambda=$ $s$ and even lower for $\lambda=s / 10$ or $\lambda=10 s$; this clearly demonstrates how useful it is to calculate $R_{\mathrm{P}}$. It appears that in order for $R_{\mathrm{P}}$ to deviate less than $1 \%$, the pad should be at least 8 probe pitches wide and to deviate less than $0.1 \%$ the width must be at least 20 probe pitches. Note, when $\lambda$ is comparable to $s$, the behavior of four-point probe MTJ measurements differs from similar measurements on a single sheet, where measurements performed on a mirror symmetry line of a small sample (such as a square) results in $R_{\mathrm{P}}$ exactly identical to that of an infinite sheet. ${ }^{19}$ However, when $\lambda$ is either much smaller or much larger than $s$, the sample behaves like a single sheet and the relative deviation between measurement on a symmetry line of a pad and on an infinite sample will vanish.

\section{EXPERIMENTAL}

The measured sample was a $200 \mathrm{~mm}$ silicon wafer with a patterned, in-plane (bottom electrode)/PtMn $(16 \mathrm{~nm}) /$ $\mathrm{Co}_{70} \mathrm{Fe}_{30}(2.2 \mathrm{~nm}) / \mathrm{Ru}(0.85 \mathrm{~nm}) / \mathrm{Co}_{40} \mathrm{Fe}_{40} \mathrm{~B}_{20}(2.5 \mathrm{~nm}) / \mathrm{MgO}$ $(1 \mathrm{~nm}) / \mathrm{Co}_{40} \mathrm{Fe}_{40} \mathrm{~B}_{20}(2.5 \mathrm{~nm}) /($ top electrode $)$ MTJ prepared using magnetron sputtering processes with a subsequent thermal anneal. The bottom electrode comprised Ta $(5 \mathrm{~nm}) / \mathrm{CuN}$ $(50 \mathrm{~nm}) / \mathrm{TaN}(30 \mathrm{~nm}) / \mathrm{Ta}(5 \mathrm{~nm})$, while the top electrode comprised Ta $(5 \mathrm{~nm}) / \mathrm{Ru}(80 \mathrm{~nm})$. The stack was designed to pin the magnetization of the lower $\mathrm{CoFeB}$ layer while the magnetization of the upper $\mathrm{CoFeB}$ layer is free. In the parallel spin polarized state, the nominal transfer length of the sample is $\lambda_{\text {low }}=1.9 \mu \mathrm{m}$ while anti-parallel spin polarization results in $\lambda_{\text {high }}=3.2 \mu \mathrm{m}$. Square patterns of nominal widths $w=30,35,40,50,60,80,100,150,250,500,1000$, and $2500 \mu \mathrm{m}$ were defined on the MTJ wafer using photolithography and ion beam etching.

Measurements were done on a semi-automatic CAPRES CIPTech-M200 using a micro 12-point probe as shown in Fig. 4. For each measurement, a sub-probe with only 4 electrodes was used. This allows for measurements with different probe pitches without replacement or re-engagement of the probe between measurements. In a measurement series, eight different sub-probes were used. Only some of the eight subprobes are equidistant, thus the average probe pitch $\langle s\rangle$ is reported in each case; the average probe pitch of the eight sub-probes varies from $\langle s\rangle=1.5 \mu \mathrm{m}$ to $\langle s\rangle=8.3 \mu \mathrm{m}$. Details of the probe design can be found in Ref. 16. Nonequidistant probes are accounted for in the theoretical models, and thus, this fact will not affect the results.

Two series of CIPT measurements were performed with the eight sub-probes: a series with an applied magnetic flux density $\left(B_{x}=-15 \mathrm{mT}\right)$ which results in the low resistance 


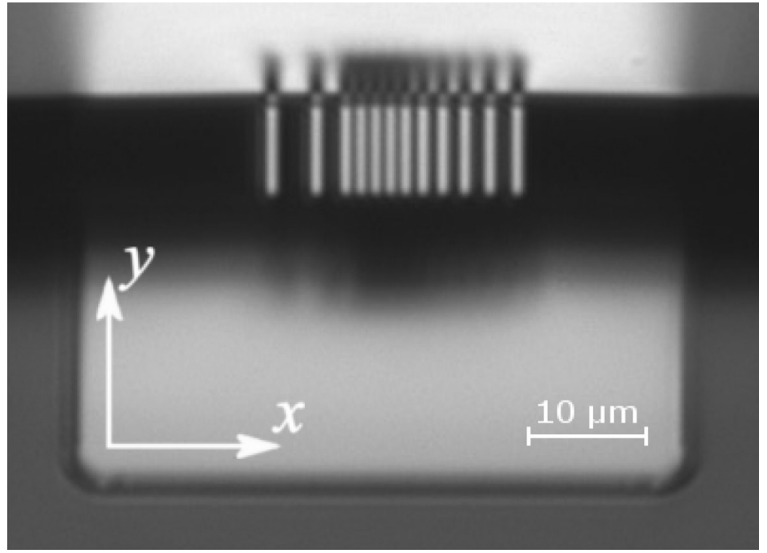

FIG. 4. Picture of a micro 12-point probe positioned over a $w=50 \mu \mathrm{m}$ pad.

parallel spin state corresponding to $\mathrm{RA}_{\text {low }}$, and a second series with an applied magnetic flux density $\left(B_{x}=15 \mathrm{mT}\right)$ which results in the high resistance anti-parallel spin state corresponding to $\mathrm{RA}_{\text {high. }}$ For each sub-probe and field direction, both $\mathrm{A}$ and $\mathrm{B}$ configuration measurements were done. From the $R_{\mathrm{A}}$ and $R_{\mathrm{B}}$ pairs, $16 R_{\mathrm{P}}$ values were calculated and used for least square fitting of the CIPT models for both infinite samples and rectangular samples, respectively. From the fits, the model parameters (e.g., $R_{\mathrm{t}}, R_{\mathrm{b}}, \mathrm{RA}_{\text {low }}, \mathrm{RA}_{\text {high }}$ ) were extracted.

CIPT measurements were performed on square pads with 12 different widths ranging from $30 \mu \mathrm{m}$ to $2.5 \mathrm{~mm}$ with the probe positioned at the centre of the pads and the line of the probe parallel to an edge of the pads. Four different pads of each size were measured, and two measurements were performed on each pad, i.e., 8 measurements were performed for each of the 12 different sized squares. Since $R_{\mathrm{t}}$ and $R_{\mathrm{b}}$ do not depend on RA, they are assumed to be independent on the magnetic field direction. This leaves $R_{\mathrm{t}}, R_{\mathrm{b}}, \mathrm{RA}_{\text {low }}$, and $\mathrm{RA}_{\text {high }}$ as the four free fitting parameters assuming that the 12-point probe is placed exactly at the centre of the pads and that the dimensions of the pads are accurately known.

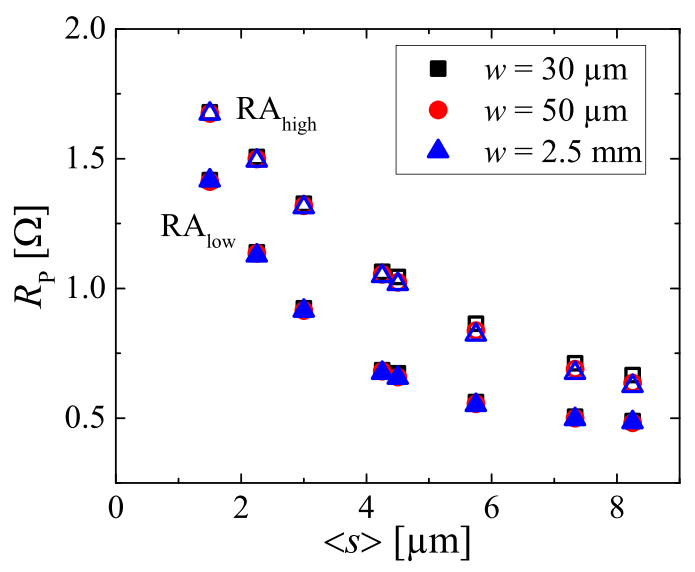

FIG. 5. Measured pseudo sheet resistances $R_{\mathrm{P}}$ as function of average probe pitch for squares with widths of $30 \mu \mathrm{m}, 50 \mu \mathrm{m}$, and $2.5 \mathrm{~mm}$. Closed symbols signify measurements with $B_{x}=-15 \mathrm{mT}$ ( $\left.\mathrm{RA}_{\mathrm{low}}\right)$, while open symbols signify measurements with $B_{x}=15 \mathrm{mT}\left(\mathrm{RA}_{\mathrm{high}}\right)$.

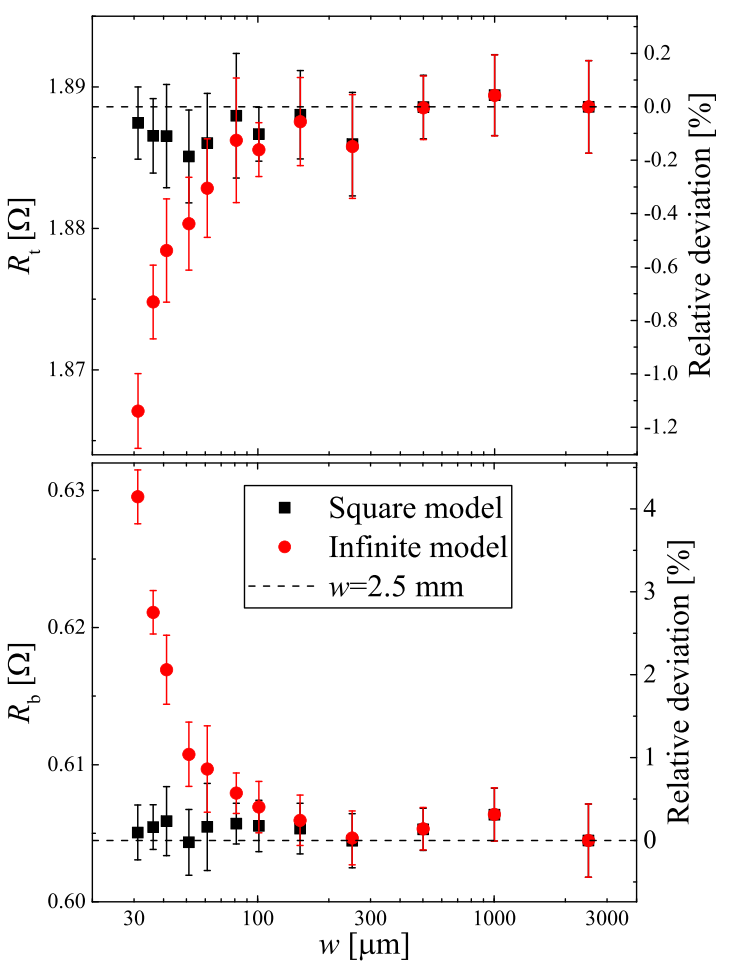

FIG. 6. Extracted values of $R_{\mathrm{t}}$ and $R_{\mathrm{b}}$ as function of sample width. Both the infinite sample CIPT model and the square sample CIPT model were fitted to the measurements. The dashed lines are mean values obtained on the $2.5 \mathrm{~mm}$ pads.

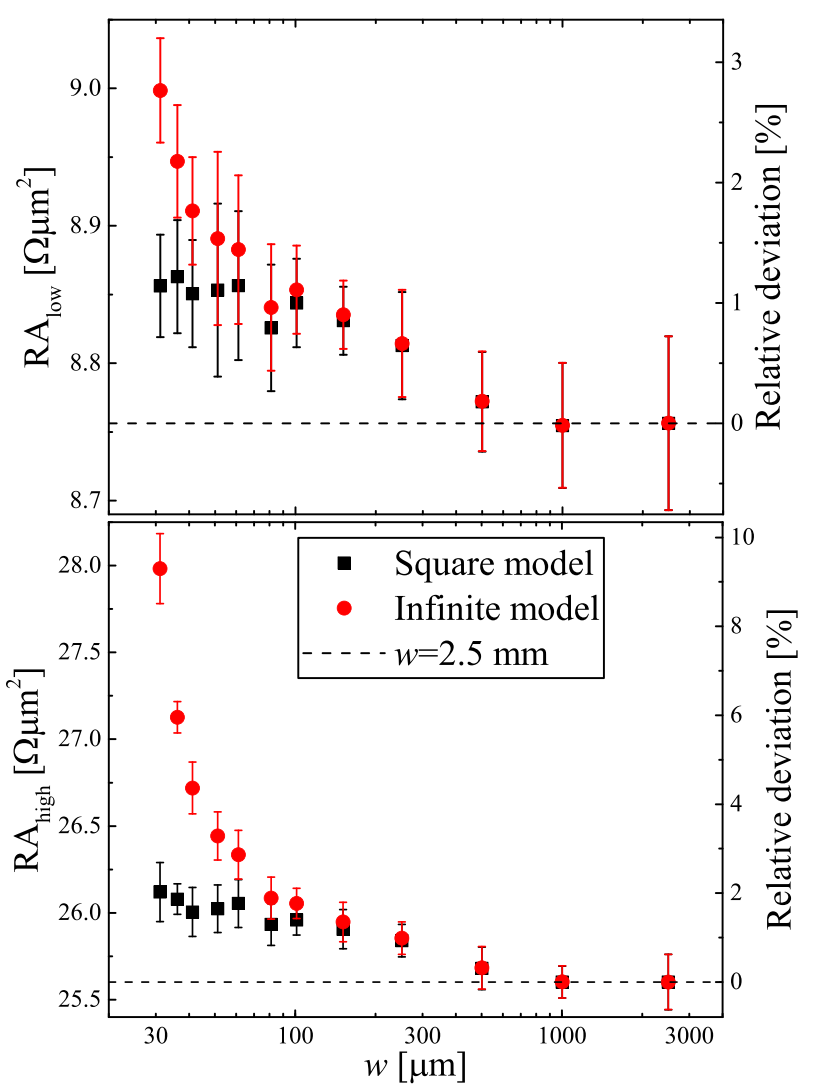

FIG. 7. Extracted values of $\mathrm{RA}_{\text {low }}$ and $\mathrm{RA}_{\text {high }}$ as function of sample width. Both the infinite sample CIPT model and the square sample CIPT model were fitted to the measurements. The dashed lines are mean values obtained on the $2.5 \mathrm{~mm}$ pads. 
CIPT measurements were also performed as function of $y$-position on a $30 \mu \mathrm{m}$ square pad (again with the line of the probe parallel to an edge of the pad). When the model was fitted to these measurements $R_{\mathrm{t}}, R_{\mathrm{b}}, \mathrm{RA}_{\text {low }}, \mathrm{RA}_{\text {high }}, x_{c}$, and $y_{c}$ were used as free fitting parameters. Thus, only the dimension of the pad was assumed known.

\section{RESULTS AND DISCUSSION}

\section{A. Measurements on various square pads}

In Fig. 5, the measured pseudo sheet resistances for the eight sub-probes are plotted as function of average probe pitch for measurements on the pads with widths of $30 \mu \mathrm{m}$, $50 \mu \mathrm{m}$, and $2.5 \mathrm{~mm}$. As expected, the measured $R_{\mathrm{P}}$ increases with decreasing probe pitch, while the measured resistances are largely independent on the pad size, and only resistances measured with large pitch on the small samples deviate a few percent from that on the large pad, this is, in agreement with Fig. 3. Figure 5 also represents an example of the data to which the two theoretical CIPT models are fitted.

By fitting CIPT models for an infinite sample and a square sample to the measured $R_{\mathrm{P}}$ data, the electrical parameters $\left(R_{\mathrm{t}}, R_{\mathrm{b}}, \mathrm{RA}_{\text {low }}\right.$, and $\left.\mathrm{RA}_{\text {high }}\right)$ of the stack are extracted as function of sample size. In Figs. 6 and $7, R_{\mathrm{t}}, R_{\mathrm{b}}, \mathrm{RA}_{\text {low }}$, and $\mathrm{RA}_{\text {high }}$ are plotted as function of samples size. The values extracted from both of the CIPT models are plotted for each parameter. The error bars correspond to one standard deviation. The horizontal dashed lines represent the mean values obtained from measurements on the $2.5 \mathrm{~mm}$ pads. The $2.5 \mathrm{~mm}$ pads are so large compared to the probe pitches used in this study that measurements may be taken as originating from an infinite sample, thus the mean of these values are used to define the zero of the relative deviation on the right $y$-axis.

The parameter values extracted from the two models essentially coincide for samples larger than $100 \mu \mathrm{m}$. For samples smaller than $100 \mu \mathrm{m}$, the values obtained from the model for an infinite sample start to deviate from the level obtained for large samples. These trends are in good agreement with the theoretical deviations plotted in Fig. 3. Since the largest probe pitch used is $8.3 \mu \mathrm{m}, w / s$ will become less than 12 for samples smaller than $100 \mu \mathrm{m}$, which leads to an overestimation of $R_{\mathrm{P}}$ by approximately $0.5 \%$. As the sample size is decreased, this overestimation along with the overestimation of the resistances for the other sub-probes will increase. Thus, the extracted parameter results become increasingly different from parameters from measurements on a large pad.

From Fig. 6, it is seen that the values of $R_{\mathrm{t}}$ and $R_{\mathrm{b}}$ obtained using the model for a square sample are independent of sample size. The values of $\mathrm{RA}_{\text {low }}$ and $\mathrm{RA}_{\text {high }}$ (Fig. 7) change slightly as a function of sample size. These small changes are believed to be due to sample variation across the wafer. Note, the resistance-area product is known to depend exponentially on the tunnel barrier thickness, ${ }^{20}$ and thus the observed variation in resistance-area product may be caused by deep sub-nanometer variations in tunnel barrier thickness. In contrast, the infinite sample CIPT model results in extracted parameters that deviate significantly when large and small samples are compared.

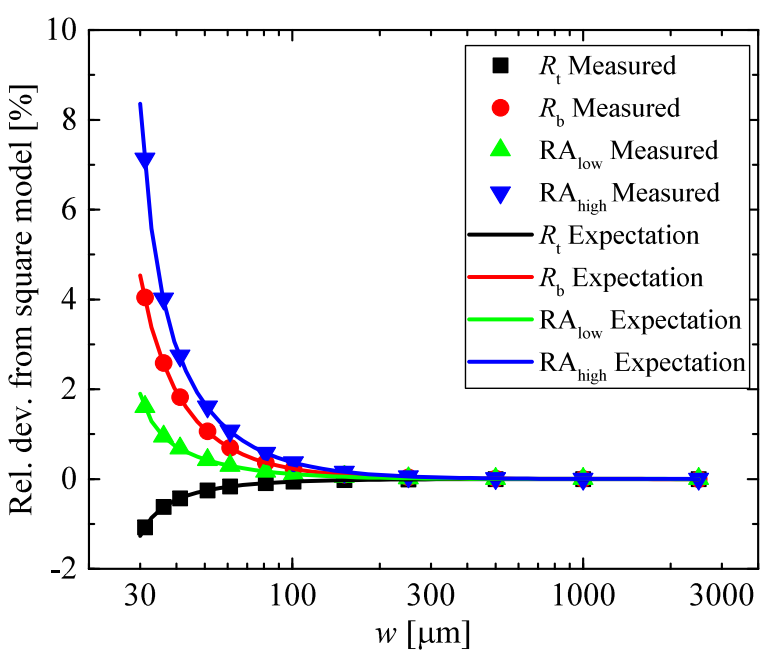

FIG. 8. Relative deviation between sample parameters extracted from fits with the square and infinite models to measurements, respectively. The solid lines are expected relative deviations calculated from fits to synthetic data.

Figure 8 shows the point by point relative deviation between sample parameters extracted using the square and infinite models, respectively. The full curves in Fig. 8 are obtained using the same procedure on synthetic CIPT data calculated using sample parameters obtained from the $2.5 \mathrm{~mm}$ sample. The near perfect agreement between real data (points) and the synthetic calculations suggests that the slight increase in extracted RA values (Fig. 7) for smaller pads is probably due to real sample variations.

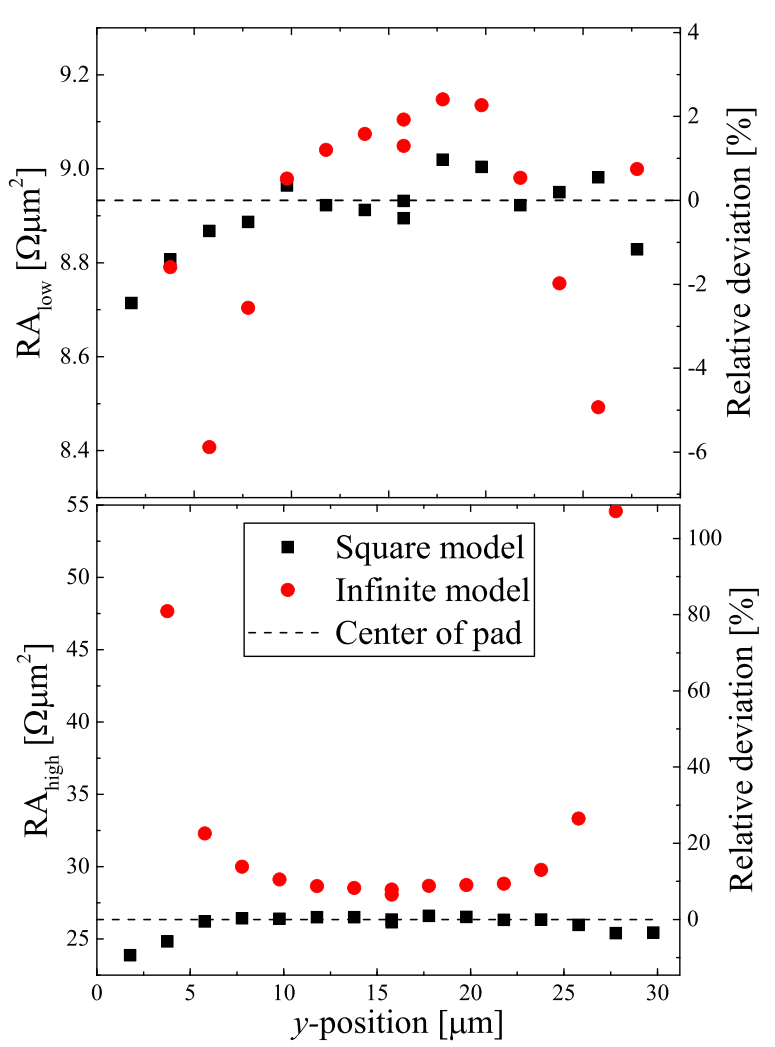

FIG. 9. Extracted values of $\mathrm{RA}_{\text {low }}$ (top) and $\mathrm{RA}_{\text {high }}$ (bottom) as function of $y$-position on a $30 \mu \mathrm{m}$ pad. 


\section{B. Sensitivity to probe position on a small pad}

Figure 9 shows $\mathrm{RA}_{\text {low }}$ and $\mathrm{RA}_{\text {high }}$ extracted from CIPT measurements on a $30 \mu \mathrm{m} \times 30 \mu \mathrm{m}$ square pad as function of probe position when the probe was scanned parallel to an edge of the pad. The horizontal dashed lines represent the RA values obtained at the centre of the pad; they are also used to define the reference for relative deviation shown on the right axes. From the plots, it is seen that $\mathrm{RA}_{\text {low }}$ and $\mathrm{RA}_{\text {high }}$ can be extracted within $\pm 1 \%$ as long as the probe is more than $5 \mu \mathrm{m}$ from the sample boundary if the square sample CIPT model is used. The reduced RA values extracted with the square model from measurements closer to the boundary may be due to sample artifacts. The ion beam etching used to define the pads may cause some radiation damage or modification of the tunnel barrier in vicinity of the edge, and, in addition, material redeposited during etching may cause a leakage current path across the tunnel junction at the edges; this would result in an apparent reduction in RA for measurements done in close proximity to the edge as observed experimentally, whereas the sheet resistances should not be affected in agreement with experiments.

Use of the infinite sample CIPT model leads to larger discrepancies; if the probe is near the centre of the pad, RA is overestimated systematically and close to the boundary the error becomes very large.

The finite size of the electrode-sample contact area is known to be a potential source of error. ${ }^{21}$ For the conditions used in this study, the contact diameter is on the order of $100 \mathrm{~nm}$ while the smallest electrode pitch is $1.5 \mu \mathrm{m}$, and thus, the ratio of pitch to contact diameter is about 15; this ratio is so large that according to Ilse et al. ${ }^{21}$ virtually, no measurable effect of the finite contact size is expected.

From the classical CIPT measurements on infinite samples, it is known that sample parameters can be extracted reliably if the smallest electrode pitch is of the same order of magnitude as $\lambda$; i.e., it is essential that at least some of the measurements are done in a regime where the measured resistances deviate from single sheet behavior. Based on the presented results, we are confident that if the minimum electrode pitch is comparable to the transfer length, $\lambda \sim s$, it will always be possible to extract sample parameters from measurements on a square pad with a minimum size just slightly larger than the total probe width such that the probe can be accommodated on the pad.

\section{CONCLUSION}

We have shown that CIPT measurement can indeed be performed on small MTJ square pads and that meaningful, accurate sample parameters can be extracted from the measurements as long as $\lambda \sim s$. Furthermore, it is shown that if the CIPT measurements are performed at the center of the pad and the width of the square is 20 times larger than $\lambda$ and 12 times larger than the largest probe pitch, it does not matter whether the sample is treated as a rectangle or an infinite sample. However, for smaller sample sizes, the new model for a rectangular sample should be used to obtain reliable results. We have demonstrated successful CIPT measurements on square samples almost as small as the total probe width. Measurements on such small samples show that RA can be measured within $\pm 1 \%$ as long as the probe lands more than $5 \mu \mathrm{m}$ from the boundary parallel to the probe pins; in practice, this means that even on such a small pad the sensitivity to probe misalignment is small. The results are very promising and may lead to application of CIPT measurements at later stages in MTJ fabrication as well as on pads in scribe lines on fabrication wafers. A similar method to the CIPT method may also be applied to other multi layer structures without changing the magnetic field, ${ }^{15}$ for instance, this method can also be used to characterize leakage current ${ }^{22}$ and the specific contact resistance between thin films. ${ }^{15}$ However, the probe pitches may need to be adjusted to the characteristic length $\lambda$ of such a sample.

\section{ACKNOWLEDGMENTS}

This work was financially supported by Innovation Fund Denmark. CINF is funded by the Danish National Research Foundation (DNRF54). This work was supported by Villum Foundation, project no. VKR023117.

\section{APPENDIX: $\boldsymbol{R}_{\mathrm{P}}$ VS. SAMPLE SIZE}

Here, we show contour plots to illustrate the general behavior of the pseudo sheet resistance when sample and probe parameters are varied.

Figure 10 shows three contour plots of the relative deviation of the pseudo sheet resistance calculated at the center of a pad compared to that of an infinite sample as a function
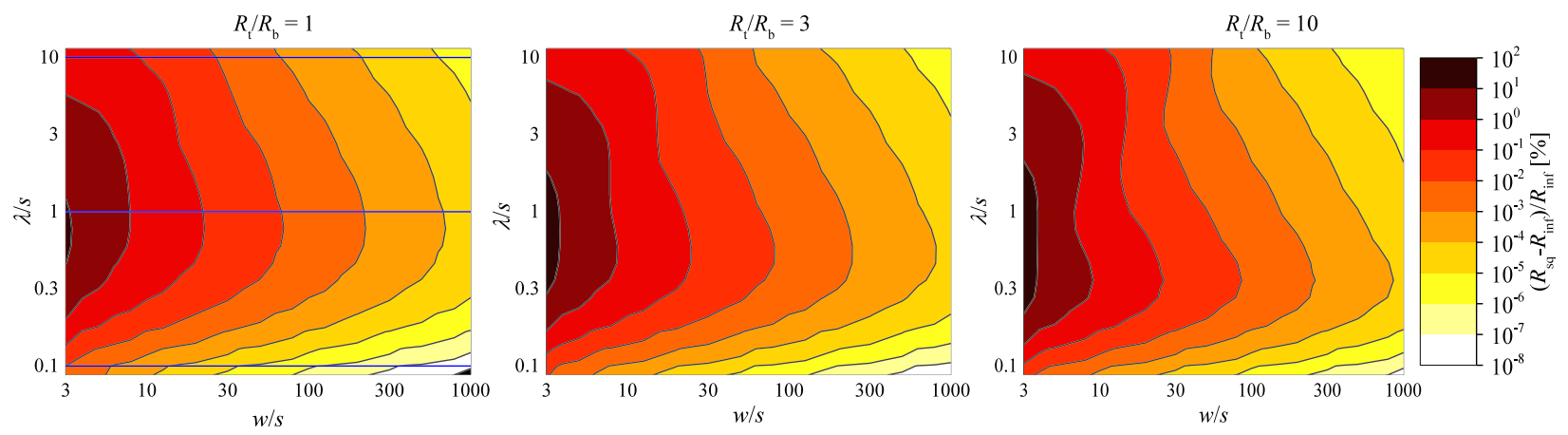

FIG. 10. Relative deviation of the pseudo sheet resistance as function of $w / s$ and $\lambda / s$ with the ratio $R_{\mathrm{t}} / R_{\mathrm{b}}$ as parameter; i.e., $R_{\mathrm{t}} / R_{\mathrm{b}}=1$ (left), $R_{\mathrm{t}} / R_{\mathrm{b}}=3$ (centre), and $R_{\mathrm{t}} / R_{\mathrm{b}}=10$ (right). The blue horizontal lines in the subplot for $R_{\mathrm{t}} / R_{\mathrm{b}}=1$ show the traces plotted in Fig. 3 . 
of $w / s$ and $\lambda / s$, with the ratio $R_{\mathrm{t}} / R_{\mathrm{b}}=1,3$ and 10 as parameter. In the subplot with $R_{\mathrm{t}} / R_{\mathrm{b}}=1$, the blue horizontal lines show the traces plotted in Fig. 3.

Figure 10 shows that in general the relative deviation decreases with $w / s$. Furthermore, the relative deviation has a local maximum near $\lambda \sim s$; the local maximum shifts slightly towards lower values of $\lambda / s$ as $R_{\mathrm{t}} / R_{\mathrm{b}}$ is increased. In the extreme cases of $\lambda \ll s$ and $\lambda \gg s$, the relative deviation approaches zero. This is expected, since position corrected four-point measurements on the sample show single sheet behavior in these cases, and for single sheet samples, the pseudo sheet resistance measured on a symmetry line on a pad is identical to that measured on an infinite sample. ${ }^{19}$

${ }^{1}$ M. Julliere, Phys. Lett. A 54, 225 (1975).

${ }^{2}$ R. Ferreira, P. Wisniowski, P. P. Freitas, J. Langer, B. Ocker, and W. Maass, J. Appl. Phys. 99, 08K706 (2006).

${ }^{3}$ S. Mao, J. Nowak, D. Song, P. Kolbo, L. Wang, E. Linville, D. Saunders, E. Murdock, and P. Ryan, IEEE Trans. Magn. 38, 78 (2002).

${ }^{4}$ J.-G. J. Zhu and C. Park, Mater. Today 9, 36 (2006).

${ }^{5}$ T. Kagami, T. Kuwashima, S. Miura, T. Uesugi, K. Barada, N. Ohta, N. Kasahara, K. Sato, T. Kanaya, H. Kiyono et al., IEEE Trans. Magn. 42, 93 (2006).

${ }^{6}$ S. Araki, K. Sato, T. Kagami, S. Saruki, T. Uesugi, N. Kasahara, T. Kuwashima, N. Ohta, J. Sun, K. Nagai et al., IEEE Trans. Magn. 38, 72 (2002).

${ }^{7}$ M. K. Ho, C. H. Tsang, R. E. Fontana, Jr., S. S. Parkin, K. J. Carey, T. Pan, S. MacDonald, P. C. Arnett, and J. O. Moore, IEEE Trans. Magn. 37, 1691 (2001).
${ }^{8}$ R. W. Dave, G. Steiner, J. Slaughter, J. Sun, B. Craigo, S. Pietambaram, K. Smith, G. Grynkewich, M. DeHerrera, J. Akerman et al., IEEE Trans. Magn. 42, 1935 (2006).

${ }^{9}$ B. N. Engel, N. D. Rizzo, J. Janesky, J. M. Slaughter, R. Dave, M. DeHerrera, M. Durlam, and S. Tehrani, IEEE Trans. Nanotechnol. 1, 32 (2002).

${ }^{10}$ N. Rizzo, D. Houssameddine, J. Janesky, R. Whig, F. Mancoff, M. Schneider, M. DeHerrera, J. Sun, K. Nagel, S. Deshpande et al., IEEE Trans. Magn. 49, 4441 (2013).

${ }^{11}$ S. Parkin, K. Roche, M. Samant, P. Rice, R. Beyers, R. Scheuerlein, E. Osullivan, S. Brown, J. Bucchigano, D. Abraham et al., J. Appl. Phys. 85, 5828 (1999).

${ }^{12}$ D. C. Worledge and P. L. Trouilloud, Appl. Phys. Lett. 83, 84 (2003).

${ }^{13}$ S. Ramo, J. R. Whinnery, and T. Van Duzer, Fields and Waves in Communication Electronics (Wiley, New York, 1984).

${ }^{14}$ D. H. Petersen, O. Hansen, R. Lin, and P. F. Nielsen, J. Appl. Phys. 104, 013710 (2008).

${ }^{15}$ Q. T. Vu, E. Kolawa, L. Halperin, and M.-A. Nicolet, Solid State Electron. 34, 279 (1991).

${ }^{16}$ D. Kjaer, O. Hansen, F. W. Østerberg, H. H. Henrichsen, C. Markvardsen, P. F. Nielsen, and D. H. Petersen, Meas. Sci. Technol. 26, 095005 (2015).

${ }^{17}$ L. J. Van der Pauw, Philips Tech. Rev. 20, 220 (1958).

${ }^{18}$ L. J. Van der Pauw, Philips. Res. Rep. 13, 1 (1958).

${ }^{19}$ S. Thorsteinsson, F. Wang, D. H. Petersen, T. M. Hansen, D. Kjær, R. Lin, J.-Y. Kim, P. F. Nielsen, and O. Hansen, Rev. Sci. Instrum. 80, 053902 (2009).

${ }^{20}$ S. Yuasa, T. Nagahama, A. Fukushima, Y. Suzuki, and K. Ando, Nat. Mater. 3, 868 (2004).

${ }^{21}$ K. Ilse, T. Tänzer, C. Hagendorf, and M. Turek, J. Appl. Phys. 116, 224509 (2014).

${ }^{22}$ R. Lin, D. H. Petersen, F. Wang, B. R. Yates, K. S. Jones, O. Hansen, A. Kontos, and P. F. Nielsen, AIP Conf. Proc. 1496, 175 (2012). 